\title{
ON PENDENT DROPS IN A CAPILLARY TUBE
}

\author{
G. HUI SKEN
}

The energy functional for a pendent drop in a capillary tube is neither convex nor bounded from below. We obtain local minima of the energy by making the physically reasonable assumption that the gravitation or the prescribed volume of the drop is small.

\section{Introduction}

We consider a drop of prescribed volume $\dot{V}$ hanging from inside the top of an upside down capillary tube. Let the bounded domain $\Omega \subset \mathbf{R}^{n}$, $n \geq 2$, denote the cross-section of the tube and assume that the top of the tube and the surface of the drop can be represented as graphs of functions $\psi_{1}$ and $u$ on $\Omega$. For convenience of notation it is preferable to make a change of the coordinate system, that is, we reverse the cylinder and assume the gravitation to be directed upward. Then $\psi_{1}$ becomes the bottom of the tube and we have

$$
u \geq \psi_{1}, \quad v=\int_{\Omega} u-\psi_{1} d x
$$

Now the physical principle of virtual work leads to the variational problem

$$
\begin{aligned}
E(v) & =\int_{\Omega}\left(1+|D v|^{2}\right)^{\frac{1}{2}} d x-\frac{\kappa}{2} \int_{\Omega} v^{2} d x+\int_{\partial \Omega} B v d H n-1 \\
& \rightarrow \min \text { in } K
\end{aligned}
$$

Received 18 July 1983.

Copyright Clearance Centre, Inc. Serial-fee code: 0004-9727/83 $\$$ A2.00 + 0.00 . 
where $K$ is some suitable convex function space. Here $\kappa \geq 0$ is the capillarity constant and $\beta \in L^{\infty}(\partial \Omega)$ is the cosine of the contact angle between $u$ and the cylinder walls. $E$ represents the energy of our system and we have a minus sign in front of $k$ since the gravitation is upward directed. We assumed that there is no contribution to the energy from the bottom of the tube, that is that the liquid will 'wet' the obstacle $\psi_{1}$.

A natural choice of $K$ would be

$$
K_{1}=H^{l, \infty}(\Omega) \cap\left\{v \geq \psi_{1}\right\} \cap\left\{\int_{\Omega} v-\psi_{1} d x=v\right\}
$$

and in the case of downward directed gravitation the corresponding variational problem was solved by Gerhardt in [2], [4]. In our case it is not possible to minimize $E$ in $K_{1}$ since the energy is not bounded from below on this set. However, it was shown in [6], that the corresponding variational inequality to $(0.2)$ admits a solution under the physically reasonable assumption that the capillarity constant $K$ or the volume $V$ is small enough (see [6] or Chapter 2 below). If we consider these solutions as critical points of the energy functional, the question arises whether there are critical points which actually minimize the energy at least compared with functions which are not too far away.

To make the terminology 'not too far away' precise, we introduce the sets

$$
\text { (0.4) } K_{2}=B V(\Omega) \cap\left\{\psi_{1} \leq v \leq \psi_{2}\right\} \cap\left\{\int_{\Omega} v-\psi_{1} d x=V\right\}
$$

and

$$
\text { (0.5) } K_{3}=H^{l, \infty}(\Omega) \cap\left\{\psi_{1} \leq v \leq \psi_{2}\right\} \cap\left\{\int_{\Omega} v-\psi_{1} d x=V\right\} \cap\left\{|D v|_{\infty} \leq N\right\}
$$

where $\psi_{2}>\psi_{1}$ is a second obstacle and $N$ is some large constant. To ensure that these sets are not empty, we have to assume

$$
\int_{\Omega} \psi_{2}-\psi_{1} d x \geq V
$$

Assuming suitable smoothness of the data we can show that the minimizing problem (0.2) can be solved in $K_{2}$ and $k_{3}$ for any $\kappa$ and $V$. 
Moreover, if $N$ and $\psi_{2}$ are large enough we can ensure that for suitable small $K$ or $V$ the constraints $\psi_{2}$ and $N$ are not reached by the solution. These solutions can then be considered as hanging drops, which minimize the energy in $K_{2}$ respectively $K_{3}$. Furthermore, a minimum of $E$ in $K_{3}$, which is strictly away from the constraints $\psi_{2}$ and $N$, satisfies a variational inequality as in [6] and has therefore all the regularity properties established in [6] and [7].

Giusti [5] used a second obstacle to show the existence of pendent drops in a different setting.

Notation. We denote by $H^{m, p}(\Omega)$ the Sobolev spaces and by $B V(\Omega)$ the space of functions of bounded variation. $H_{n}$ is the $n$-dimensional Hausdorff measure and $|\cdot|_{\infty}$ the supremum norm on $\Omega$. We shall denote by $c=c(\ldots)$ various constants, whereas indices will be used if a constant recurs at another place.

\section{Minima in $B V(\Omega)$}

From now on we consider the more general energy functional

(1.1) $E(v)=\int_{\Omega}\left(1+|D v|^{2}\right)^{\frac{1}{2}} d x+\int_{\Omega} \int_{0}^{v} H(x, t) d t d x-\frac{\kappa}{2} \int_{\Omega} v^{2} d x+\int_{\partial \Omega} \beta v d H n-1$ where

$$
B \in L^{\infty}(\partial \Omega), \quad H \in C^{O, 1}\left(\mathbf{R}^{n} \times \mathbf{R}\right)
$$

satisfy

$$
|B| \leq 1-a, a>0 \text {, }
$$

and

$$
\text { (1.4) } \frac{\partial H}{\partial t} \geq 0, \sup _{\Omega} H(x, t) \leq \alpha(1+t), \alpha>0 \text {, for all } t>0 \text {. }
$$

We assume that $\Omega \subset \mathbb{R}^{n}, n \geq 2$, is a bounded domain with Lipschitz boundary $\partial \Omega$ satisfying an interior sphere condition of radius $R$.

Here $\Omega$ is said to satisfy an ISC of radius $R$, if for any boundary 
point $x_{0} \in \partial \Omega$ there exists a ball $B$ of radius $R$ such that $B \subset \Omega$ and $x_{0} \in \bar{B}$.

This interior sphere condition is imposed in view of the following result (see [3], Remark 2).

LEMMA 1. Let $\Omega$ be as above; then

(1.5) $\int_{\partial \Omega}|v| d H_{n-1} \leq \int_{\Omega_{\varepsilon}}|D v| d x+c_{\varepsilon} \cdot \int_{\Omega}|v| d x$ for alz $v \in B V(\Omega)$

where $\Omega_{\varepsilon}=\{x \in \Omega \mid \operatorname{dist}(x, \partial \Omega)<\varepsilon\}, c_{\varepsilon}$ depends on $\varepsilon, R, \partial \Omega$ and $0<\varepsilon \leq R / 2$ is arbitrary.

Then, assuming all these conditions to hold, we have

THEOREM 1. Let $\psi_{i} \in C^{0, I}(\bar{\Omega}), i=1,2$. Then the minimizing probzem

$$
E(v) \rightarrow \min \text { in } K_{2}
$$

has a solution $u$ for any $k$ and $V$, if (0.6) holds.

Proof. It is obvious that

$$
E(v) \geq-C \text { for all } v \in K_{2}
$$

for some constant $C$, since $v$ is between the two bounded functions $\psi_{1}$ and $\Psi_{2}$. Now let $v_{\eta}$ be a minimizing sequence of the variational problem (1.6). If we define

$$
w=\psi_{1}+V \cdot\left(\psi_{2}-\psi_{1}\right) /\left(\int_{\Omega} \psi_{2}-\psi_{1} d x\right)
$$

then $w \in K_{2}$ in view of $(0.6)$ and eventually we have

$$
E\left(v_{2}\right) \leq E(w)
$$

Again using $\psi_{1} \leq v \leq \Psi_{2}$ we conclude

$$
\int_{\Omega}\left|D v_{\eta}\right| d x \leq \int_{\Omega}\left(1+\left|D v_{\eta}\right|^{2}\right)^{\frac{1}{2}} d x \leq E(w)+C
$$


where the constant depends on $\left|\psi_{2}\right|_{\infty}, \kappa, \Omega, \partial \Omega$ and $\left|H\left(\cdot, \psi_{i}\right\}\right|_{\infty}$, $i=1,2$. Thus, the sequence

$$
\int_{\Omega}\left|D v_{\eta}\right| d x+\int_{\Omega}\left|v_{\eta}\right| d x
$$

is uniformly bounded. Then, as in ([2], Theorem 2.1) we can find a subsequence of the $v_{l}$ converging in $L^{1}(\Omega)$ to some function $u \in B V(\Omega)$.

It was also shown in [2], that the expression

$$
\int_{\Omega}\left(1+|D v|^{2}\right)^{\frac{1}{2}} d x+\int_{\Omega} \int_{0}^{v} H(x, t) d t d x+\int_{\partial \Omega} B v d H n-1 .
$$

is lower semicontinuous with respect to this convergence. To handle the term

$$
-\frac{\kappa}{2} \int_{\Omega} v_{2}^{2} d x
$$

we observe that in view of $\psi_{1} \leq v_{2} \leq \psi_{2}$ the Lebesgue dominated convergence theorem can be applied. Therefore

$$
E(u) \leq \lim \inf E\left(v_{\eta}\right)
$$

and $u$ is the desired solution.

THEOREM 2. If we choose

$$
\psi_{2}=\psi_{1}+M, M>0,
$$

then

(i) for arbitrary but fixed $k$ and $M$ we can find $V_{0}>0$, such that for any $0<v<V_{0}$ the solution $u$ of (1.6) lies strictly below $\psi_{2}$;

(ii) for $M$ large enough and $V$ or $k$ small enough the solution $u$ of (1.6) will be strictly below $\psi_{2}$.

Proof. The conclusion of the theorem follows from the estimate

$$
u-\psi_{1} \leq c_{1} \cdot\left(c_{2}+k \cdot\left|\psi_{2}\right|_{\infty}\right) \cdot v^{1 /(n+1)}
$$


where the constants depend on $H, n, a, \Omega, \partial \Omega,\left|\psi_{1}\right|_{\infty}$ and $\left|D \psi_{1}\right|_{\infty}$. To establish the estimate, we choose the test function

$$
v=\psi_{1}+\min \left(u-\psi_{1}, k\right)+\frac{1}{|\Omega|} \cdot \int_{\Omega} \max \left(u-\psi_{1}-k, 0\right)
$$

for any $0 \leq k \leq M$. Since

$$
\min \left(u-\psi_{1}, k\right)+\max \left(u-\psi_{1}-k, 0\right)=u-\psi_{1}
$$

we have $\int_{\Omega} v-\psi_{1} d x=V$. The estimate

$$
\psi_{1} \leq v \leq \psi_{2}
$$

follows from $0 \leq k \leq M$ and the fact that $u \in K_{2}$. Therefore $v \in K_{2}$ and

$$
E(u) \leq E(v)
$$

We obtain

$$
\text { (1.2I) } \begin{aligned}
& \int_{\Omega}\left(1+|D u|^{2}\right)^{\frac{1}{2}} d x \leq \int_{\Omega}(1\left.+|D v|^{2}\right)^{\frac{1}{2}} d x+\int_{\partial \Omega} \beta(v-u) d H \\
& n-1 \\
&+\frac{\kappa}{2} \cdot \int_{\Omega}(u-v)(u+v) d x+\int_{\Omega} \int_{u}^{v} H(x, t) d t d x .
\end{aligned}
$$

The first term on the right hand side can be estimated by

$$
\begin{aligned}
\int_{A(k)}\left(1+\left|D \psi_{1}\right|^{2}\right)^{\frac{1}{2}} d x+\int_{\Omega \backslash A(k)} & \left(1+|D u|^{2}\right)^{\frac{1}{2}} d x \\
& \leq 2 \cdot\left|D \psi_{1}\right|_{\infty} \cdot|A(k)|+\int_{\Omega \backslash A(k)}\left(1+|D u|^{2}\right)^{\frac{1}{2}} d x
\end{aligned}
$$

where we used the notation $A(k)=\left\{x \in \Omega \mid u-\psi_{1}>k\right\}$ and $|A(k)|=H_{n}(A(k))$. Furthermore we get from (1.3) and (1.5), (1.23) $\int_{\partial \Omega} B(v-u) d H_{n-1} \leq(1-a) \int_{\Omega}|D(v-u)| d x+e_{R / 2} \cdot \int_{\Omega}|v-u| d x$

$$
\begin{aligned}
\leq(1-a) \int_{A(k)}|D u| d x & +\left|D \psi_{1}\right|_{\infty} \cdot|A(k)| \\
& +2 \cdot c_{R / 2} \cdot \int_{A(k)} u-\psi_{1}-k d x .
\end{aligned}
$$


Moreover

(1.24) $\frac{k}{2} \cdot \int_{\Omega}(u-v)(u+v) d x \leq 2 \cdot \kappa \cdot\left|\psi_{2}\right|_{\infty} \cdot \int_{A(k)} u-\psi_{1}-k d x$.

The last term in $(1.21)$ can be estimated by

(1.25) $\left(|H(\cdot, 0)|_{\infty}+\left|H\left(\cdot, \psi_{1}\right)\right|_{\infty}+\alpha\left(1+\frac{V}{|\Omega|}+\left|\psi_{1}\right|_{\infty}\right)\right) \cdot \int_{A(k)} u-\psi_{1}-k d x$

where we used the assumptions (1.4) in a similar manner as in ([6],

Estimate 2.14). So we finally conclude, from (1.21),

(1.26) $a \cdot \int_{A(k)}|D u| d x \leq 3 \cdot\left|D \psi_{1}\right|_{\infty} \cdot|A(k)|+c \cdot \int_{A(k)} u-\psi_{1}-k d x$

where

$$
c=\left(2 \cdot k \cdot\left|\psi_{2}\right|_{\infty}+2 \cdot C_{R / 2}+|H(\cdot, 0)|_{\infty}+\left|H\left(\cdot, \psi_{1}\right)\right|_{\infty}+\alpha\left(1+\frac{V}{|\Omega|}+\left|\psi_{1}\right|_{\infty}\right)\right) .
$$

Up to a numerical constant this is exactly inequality (2.16) in [6], and the estimate (1.16) now follows in the same way as in [6].

REMARK. We can not expect the function $u$ to be regular in general, since there might be sections with vertical gradient. To avoid this possibility, we impose an additional gradient constraint in the next section.

\section{Regular minima}

In [6] we assumed that

$$
B \in C^{\mathcal{\lambda}, \alpha}(\partial \Omega), \quad H \in C^{1, \alpha}\left(\mathbf{R}^{n} \times \mathbf{R}\right)
$$

satisfy the conditions $(1.3)$ and $(1.4)$. We denoted by $A$ the minimal surface operator

$$
A u=\sum_{i=1}^{n}-D_{i}\left(a^{i}(D u)\right), a^{i}=D_{i} u /\left(1+|D u|^{2}\right)^{\frac{1}{2}}
$$

and used the notation

$$
\langle A u, \eta\rangle=\int_{\Omega} \sum_{i} a^{i} D_{i} \eta d x+\int_{\partial \Omega} B n d H_{n-1} .
$$

Then we had the following result on the existence of critical points of the 
energy functional.

THEOREM 3. Let $\Omega$ be a bounded domain of $\mathbb{R}^{n}, n \geq 2$, with boundary of class $c^{2, \alpha}$, and let the functions $\beta$ and $H$ satisfy the conditions above. Then the variational inequality

$$
\langle A u+H(x, u)-\kappa u, v-u\rangle \geq 0 \text { for alz } v \in K_{1}
$$

has a solution $u \in H^{1, \infty}(\Omega) \cap H^{2,2}(\Omega) \cap H_{10 c}^{2, \infty}(\Omega)$ if we assume $\psi_{1} \in C^{2}(\bar{\Omega})$ and if $k$ or $V$ is small enough. The solution has continuous tangential derivatives at the boundary and in the case $n=2$ we have $u \in c^{l}(\bar{\Omega})$. If we impose on $\psi_{1}$ the further condition

$$
\sum_{i}-a^{i}\left(D \psi_{1}\right) \cdot \gamma_{i} \geq B \text { on } \partial \Omega
$$

where $\gamma$ is the outer unit normal to $a \Omega$ and if $\partial \Omega \in c^{3, \alpha}$, $\beta \in C^{1,1}(\partial \Omega)$, then $u \in H^{2, \infty}(\Omega)$.

Now assume that $\Omega, \beta$ and $H$ are as in Theorem 3 and that $\psi_{2}$ has the form

$$
\psi_{2}=\psi_{1}+M, \quad \psi_{1} \in C^{2}(\bar{\Omega}), M>0 .
$$

Then we have

THEOREM 4. The minimizing problem

$$
E(v) \rightarrow \min \text { in } K_{3}
$$

has a solution $u_{0}$ for any $\kappa, V, M, N$, provided these quantities are such that $K_{3}$ is not empty.

If $N$ and $M$ are large enough and $\kappa$ or $V$ is small enough, $u_{0}$ satisfies

$$
\left|D u_{0}\right|_{\infty} \leq N-1, u_{0} \leq \psi_{2}-1
$$

Then $u_{0}$ is also a solution to the variational inequality (2.4) and the same regularity results as in Theorem 3 apply. 
REMARKS. (i) A solution $u_{0}$ satisfying (2.8) can be considered as a hanging drop which minimizes the energy in the set $K_{3}$.

(ii) $K$ and $V$ depend on $N$ and $M$ in the sense that if $N, M$ are enlarged, $k$ or $V$ has to be taken correspondingly smaller.

(iii) The proof of Theorem 4 yields an existence result for the variational inequality (2.4) without using an abstract fixed point theorem as in [6].

Proof of Theorem 4. (i) $K_{3}$ is a bounded, closed and convex set in $H^{l}, \infty(\Omega)$ and $E$ is bounded from below on $K_{3}$. It is easy to see that then some minimizing sequence tends uniformly to a solution $u_{0}$ of (2.7).

(ii) We look at the new problem

$$
E_{1}(v) \rightarrow \min \text { in } K_{1}
$$

where

(2.10) $E_{1}(v)=\int_{\Omega}\left(1+|D v|^{2}\right)^{\frac{1}{2}} d x+\int_{\Omega} \int_{0}^{v} H(x, t) d t d x$

$$
-\kappa \cdot \int_{\Omega} u_{0} \cdot v d x+\int_{\partial \Omega} \beta v d H_{n-1} .
$$

$E_{1}$ is a convex functional of the type Gerhardt considered in [2]. He minimized those functionals in the set

$$
B V(\Omega) \cap\left\{v \geq \psi_{1}\right\} \cap\left\{\int_{\Omega} v-\psi_{1} d x=v\right\}
$$

but the regularity results in [4] showed, that the solution is in $H^{1, \infty}(\Omega)$ and therefore a solution of (2.9).

Now, for the (unique) solution $u_{1}$ of (2.9) we can establish exactly the same estimate as in Theorem 2. This follows from the fact that the test function

$$
v=\psi_{1}+\min \left(u_{1}-\psi_{1}, r\right)+\frac{1}{|\Omega|} \int_{\Omega} \max \left(u_{1}-\psi_{1}-r\right) d x
$$

is in $K_{1}$ and from the inequality 


$$
u_{0} \leq \psi_{2}
$$

Thus we can choose $M$ as large and $k$ or $V$ as small that

$$
\left|u_{1}\right|_{\infty} \leq \psi_{2}-1 \text {. }
$$

In order to get a similar estimate for the gradient of $u_{1}$, we observe that $u_{1}$ also solves the variational inequality

$$
\left\langle A u_{1}+H\left(x, u_{1}\right)-K u_{0}, v-u_{1}\right\rangle \geq 0 \text { for all } v \in K_{1} \text {. }
$$

Moreover, it was shown in [2], that there is a Lagrange multiplier $\lambda \in \mathbb{R}$ such that $u_{1}$ also satisfies

(2.16) $\left\langle A u_{1}+H\left(x, u_{1}\right)-\kappa u_{0}+\lambda, v-u_{1}\right\rangle \geq 0$

$$
\text { for all } v \in K_{1}^{\prime}=H^{1, \infty}(\Omega) \cap\left\{v \geq \psi_{1}\right\} \text {. }
$$

To this variational inequality we want to apply the gradient estimate in ([6], Theorem 1.2):

"We can choose $N$ as large and $k$ or $V$ as small that

$$
\left|D u_{1}\right|_{\infty} \leq N-1 \text {, if }\left|D u_{0}\right|_{\infty} \leq N . "
$$

Actually, the gradient estimate in [6] was established for solutions of the related boundary value problem. But $u_{1}$ can be approximated by solutions of boundary value problems and it was shown in [6], that the gradient estimate for the approximating solutions is independent of the approximation parameters.

In view of (2.14), (2.15) and (2.17) the conclusion of the theorem now follows from

LEMMA 2. If $N, M, \kappa$ and $V$ are such that $u_{1} \in K_{3}$, then we have $u_{0} \equiv u_{1}$

Proof of Lemma 2. We can compute

$$
\begin{aligned}
& E_{1}\left(u_{0}\right)=E\left(u_{0}\right)-\frac{\kappa}{2} \cdot \int_{\Omega} u_{0}^{2} d x, \\
& E_{1}\left(u_{1}\right)=E\left(u_{1}\right)-\kappa \cdot \int_{\Omega} u_{0} u_{1} d x+\frac{\kappa}{2} \cdot \int_{\Omega} u_{1}^{2} d x,
\end{aligned}
$$


and therefore

$$
E_{1}\left(u_{0}\right)=E_{1}\left(u_{1}\right)+\left(E\left(u_{0}\right)-E\left(u_{1}\right)\right)-\frac{\kappa}{2} \int_{\Omega}\left|u_{0}-u_{1}\right|^{2} d x
$$

In view of the assumptions of the lemma we have

$$
E\left(u_{0}\right) \leq E\left(u_{1}\right) \text {. }
$$

Thus, if $u_{0} \neq u_{1}$ we have

$$
E_{1}\left(u_{0}\right)<E_{1}\left(u_{1}\right)
$$

a contradiction to the minimizing property of $u_{1}$.

Finally, from Lemma 4.1 in [6] we have

COROLLARY. The solution $u_{0}$ of (2.7) is zonique if $k$ is smalz enough.

\section{References}

[1] P. Concus and R. Finn, "On capillary free surfaces in a gravitational field", Acta Math. 132 (1974), 207-223.

[2] C. Gerhardt, "On the capillarity problem with constant volume", Ann. Scuola Norm. Sup. Pisa Cl. Sci. (4) 2 (1975), 304-320.

[3] C. Gerhardt, "Existence and regularity of capillary surfaces", Boll. Un. Mat. Ital. A (5) 10 (1974), 317-335.

[4] C. Gerhardt, "Global regularity of the solutions to the capillarity problem", Ann. Scuola Norm. Sup. Pisa CZ. Sci. (4) 3 (1976), $157-175$.

[5] E. Giusti, "The pendent water drop. A direct approach", Bolz. Un. Mat. Ital. A (5) 17 (1980), 458-465.

[6] Gerhard Huisken, "Capillary surfaces in negative gravitational fields" (Research Report CMA-RO7-83. Centre for Mathematical Analysis, Australian National University, Canberra, 1983). 
[7] Gerhard Huisken, "Capillary surfaces over obstacles" (Research Report CMA-RO8-83. Centre for Mathematical Analysis, Australian National University, Canberra, 1983).

Centre for Mathematical Analysis, Australian National University, GPO Box 4, Canberra, ACT 260I, Australia. 